
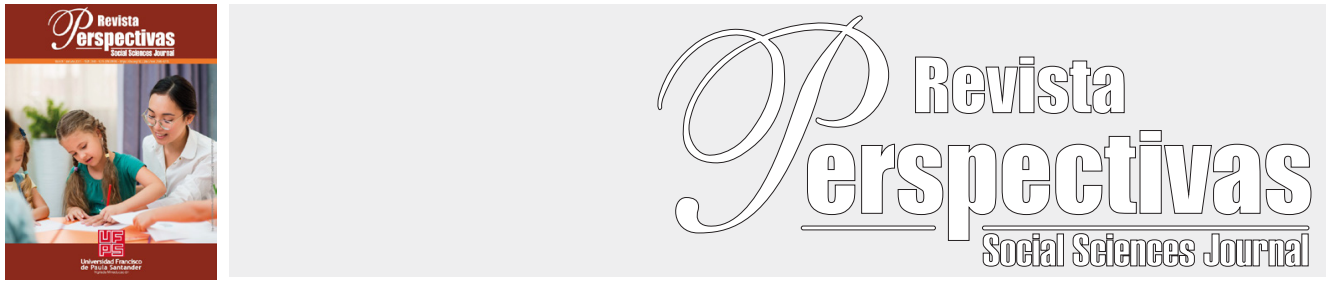

Original Article

https://doi.org/10.22463/25909215.3012

\title{
Pedagogía crítica en la formación ciudadana: caso universidad de pamplona
}

\section{Critical pedagogy in citizenship education: the case of the University of Pamplona}

\section{Giovanny Ramirez-Ayala ${ }^{1}$, Raúl Prada Núñez ${ }^{2}$}

${ }^{1}$ Especialista en Educación, Emprendimiento y Economía Solidaría, Docente Catedrático, giovanny.ramirez@unipamplona.edu.co. ORCID:0000-0001-6145-1786, Universidad de pamplona, Villa del Rosario, Colombia.

${ }^{2}$ Director Licenciatura en Educación Infantil, Facultad de Educación, Artes y Humanidades.raulprada@ufps.edu.co. ORCID: 0000-0001-61451786, Universidad Francisco de Paula Santander, Cúcuta, Colombia.

How to cite: G. Ramirez-Ayala, R. Prada-Núñez, "Pedagogía crítica en la formación ciudadana: caso universidad de pamplona.". Perspectivas, vol. 6, no. 1, pp. 85-100, 2021.

Received:August 09, 2020; Approved: November 07, 2020.

\begin{tabular}{|c|c|}
\hline & ABSTRACT \\
\hline Keywords: & $\begin{array}{l}\text { This research proposal seeks to provide an answer to the following question: if higher education projects new } \\
\text { spaces for the creation, interaction and participation of teachers and students, then how should autonomous }\end{array}$ \\
\hline $\begin{array}{l}\text { Ciudadanía; } \\
\text { cultura de paz; } \\
\text { educación superior; } \\
\text { pedagogía crítica. }\end{array}$ & $\begin{array}{l}\text { citizenship be trained from higher education at the university of pamplona ? to this end, it is planned to } \\
\text { develop curricular guidelines for training in autonomous citizenship for higher education at the university of } \\
\text { pamplona, based on representatives of this institution as well as teachers and students. it will be deepened } \\
\text { from the theoretical line of Martínez (2001) that states: it is required to train not only citizens who defend and } \\
\text { fight for the rights of the first and second generation, but also recognize the difference as a factor of progress } \\
\text { and are willing to fight to that these do not induce inequalities and injustices, even at the cost of the exercise } \\
\text { of certain levels of enjoyment of rights, by them. this proposal is a case study, the qualitative research } \\
\text { installed in the interpretive paradigm will be used as an epistemological reference, where the hermeneutical- } \\
\text { critical analysis based on the theory of symbolic interactionism prevails, supported by the theory based for } \\
\text { the construction of the findings. }\end{array}$ \\
\hline
\end{tabular}

in this order of ideas, strategies were developed that made it possible to identify citizenship training in bachelor's degree students in child pedagogy by applying techniques such as the focus group, to conclude some conclusions are drawn that show the perceptions of teachers and students regarding critical thinking.

\begin{tabular}{ll}
\hline & RESUMEN \\
\hline Palabras clave: & Esta propuesta de investigación procura facilitar una respuesta al siguiente interrogante: Si la educación superior \\
proyecta nuevos espacios de creación, interacción y participación de los docentes y estudiantes, entonces, ¿cómo & se debe formar en ciudadanía autónoma desde la educación superior en la Universidad de Pamplona? Para \\
Citizenship; & ello se proyecta elaborar lineamientos curriculares de formación en ciudadanía autónoma para la Educación \\
education; & Superior en la Universidad de Pamplona, partiendo de los representantes de esta institución tanto como de \\
critical pedagogy & los docentes y estudiantes. Se profundizará desde la línea teórica de Martínez (2001) que afirma: se exige \\
& formar no solo ciudadanos que defiendan y luchen por los derechos de primera y segunda generación, sino que \\
& también reconozcan la diferencia como factor de progreso y estén dispuestos a luchar para que estos no induzcan \\
& desigualdades e injusticias, incluso a costa del ejercicio de determinados niveles de disfrute de los derechos, por \\
& parte de ellos. Esta propuesta es un estudio de caso, se utilizará como referente epistemológico la investigación \\
& cualitativa instalada en el paradigma interpretativo, donde prima el análisis hermenéutico-crítico fundamentado \\
en la teoría del interaccionismo simbólico, apoyado en la teoría fundamentada para la construcción de los \\
hallazgos. \\
En este orden de ideas, se elaboraron estrategias que permitieron identificar la formación ciudadana en los \\
estudiantes de Licenciatura en pedagogía infantil aplicando técnicas como el grupo focal, para finalizar se \\
elaboran unas conclusiones que arrojan las percepciones de los docentes y estudiantes en cuanto al pensamiento \\
crítico.
\end{tabular}

*Corresponding author.

E-mail address: giovanny.ramirez@unipamplona.edu.co (Giovanny Ramirez-Ayala)
(c) (i) Peer review is the responsibility of the Universidad Francisco de Paula Santander.

(c) 1 This is an article under the license CC BY 4.0 


\section{Introducción}

Sin lugar a dudas, plantea Rosanvallon (2010) que la democracia reúne las distintas formas de luchas de las gentes, su emancipación y las múltiples demandas de libertad, que han caracterizado la humanidad. Hoy, la cuestión social y tecnológica, las ideologías, los movimientos de las migraciones, el sistema interconectado en el proceso de globalización que está a la vanguardia y la reconfiguración política que vive el mundo, como experiencia de la misma, presentan retos que no son fáciles de integrar, al mismo tiempo que exigen reflexiones profundas sobre el papel de los y las ciudadanas que enfrentarán las nuevas dinámicas sociales a las que asistimos y su capacidad para agenciarlas y reformarlas.

De esta manera, la educación, "en su concepción de modernidad nació para formar ciudadanos" (Magendzo, 2004, p.7), orientados hacia la productividad y la autonomía. Debido a esto, aquella debe asumir, como reto, la generación de una transformación social que aborde las nuevas condiciones sociopolíticas, económicas y culturales de la sociedad actual, a partir de la preparación de "ciudadanos capaces de lidiar, incidir y participar en dichos cambios como personas con voz ciudadana" (Magendzo, 2004, p.44). En cuanto a la responsabilidad de la Universidad en la formación de estos valores se requiere como un reto a fin de cumplir con el objetivo social de generar un capital humano que se empodere de los problemas sociales y a la vez participe y contribuya a la solución de los mismos.

Los sectores más favorecidos de nuestro mundo y en concreto los que disfrutamos del llamado "primer mundo" debemos priorizar en nuestras políticas educativas acciones orientadas a la formación de una ciudadanía activa que sea capaz de responder ante estos retos en una sociedad de la diferencia y no de la desigualdad. Esto exige formar no sólo ciudadanos que defiendan y luchen por los derechos de primera y segunda generación, sino que también reconozcan la diferencia como factor de progreso y estén dispuestos a luchar para que éstos no induzcan desigualdades e injusticias incluso a costa del ejercicio de determinados niveles de disfrute de los derechos de primera y segunda generación por parte de ellos. (Martínez 2012. p.12)

Es de anotar, que tal reflexión sobre una educación para una ciudadanía emancipada aplica en cada uno de los contextos geográficos y debe dinamizar todo tipo de lucha por la paz, la igualdad, la justicia y la libertad en cada rincón del planeta.

Estas acciones pedagógicas deben contribuir al hecho de que, en nuestro proceso de construcción personal, que no es solamente individual, sino que se da en la interacción con los otros, aprendamos a apreciar actitudes democráticas, denunciar su falta y configurar nuestra matriz personal de valores. Esta tarea educativa consiste en primer lugar en crear condiciones que fomenten la sensibilidad moral en aquellos que aprenden, a fin de constatar y vivir los conflictos morales de nuestro entorno tanto físico como mediático. Ante esta situación decía Audigier (1999):

La educación para la ciudadanía es un campo teórico y práctico donde se traducen, en acuerdos y desacuerdos, nuestras concepciones de la vida social y política, la definición de los derechos, libertades y obligación es que estimamos legítimas para nosotros y para los otros, nuestras maneras de pensar el lugar de los conflictos y de resolverlos, nuestras concepciones de la educación, del papel respectivo de la familia, de la Escuela y de otras institución es susceptibles de intervenir en la instrucción, la educación y la socialización, del lugar que tienen los conocimientos o la experiencia en la construcción de competencias.

A estos elementos se añade la necesidad de una prospectiva que permita anticipar ciertas evoluciones de nuestras sociedades. (p.6) 
A estos elementos se añade la necesidad de una prospectiva que permita anticipar ciertas evoluciones de nuestras sociedades. (p.6)

La falta de valores democráticos en la actual sociedad conlleva a la desintegración de la evolución humana, siendo esto entonces nefasto en la integralidad del desarrollo del ser humano.

En ese sentido, estas condiciones no se consiguen a través de declaraciones verbales, sistemas de enseñanza basados casi exclusivamente en la actividad del profesor o disposiciones legales que regulen los currículums de los diferentes países. Es necesario considerar que, si educar en valores es crear condiciones para conseguir todo lo que hemos dicho hasta ahora, la función reguladora y de modelaje que ejerce el profesorado es clave. Concluye: la formación de una ciudadanía activa precisa un profesorado beligerante en la defensa de principios como los apuntados y respetuoso con las distintas creencias de cada uno, formas de entender el mundo y formas de construirnos como personas, que respetando los principios de justicia enunciados conforman los diferentes modelos de vida buena de cada uno de nosotros.

Por lo tanto, esta propuesta de educar para la ciudadanía en el contexto de educación superior, de la Universidad de Pamplona, propone, propiciar los espacios donde los estudiantes puedan asumir su rol desde su responsabilidad como generaciones que a corto plazo deben construir sociedades donde se dé el dialogo, el respeto, la recuperación y la formación en valores ciudadanos. Se afirma que, la educación para la ciudadanía debe servir para reflexionar y sensibilizar sobre la convivencia y el ejercicio de la ciudadanía democrática en el centro $\mathrm{y}$ en su entorno, lo que constituye al mismo tiempo una consideración metodológica fundamental: las formas de convivencia, el respeto, el afrontamiento no violento de los conflictos, la aceptación de la diversidad y el rechazo de cualquier forma de discriminación (Martínez, 2010).
Educandos agentes generadores de cambios transformadores de una sociedad que requiere que sean partícipes para la recuperación del tejido social, que nuestra sociedad tanto lo necesita, estos nuevos desafíos, frente a los procesos de fragmentación, diferenciación y exclusión social que padecen las sociedades dificultan la construcción de una ciudadanía plena que requiere de prácticas socioeducativas que garanticen A las personas el ejercicio de un conjunto de derechos, entre ellos el de la inclusión corporación social.

La falta de valores democráticos en la actual sociedad conlleva a la desintegración de la evolución humana, siendo esto entonces nefasto en la integralidad del desarrollo del ser humano.

En ese sentido, estas condiciones no se consiguen a través de declaraciones verbales, sistemas de enseñanza basados casi exclusivamente en la actividad del profesor o disposiciones legales que regulen los currículums de los diferentes países. Es necesario considerar que, si educar en valores es crear condiciones para conseguir todo lo que hemos dicho hasta ahora, la función reguladora y de modelaje que ejerce el profesorado es clave. Concluye: la formación de una ciudadanía activa precisa un profesorado beligerante en la defensa de principios como los apuntados y respetuoso con las distintas creencias de cada uno, formas de entender el mundo y formas de construirnos como personas, que respetando los principios de justicia enunciados conforman los diferentes modelos de vida buena de cada uno de nosotros.

Por lo tanto, esta propuesta de educar para la ciudadanía en el contexto de educación superior, de la Universidad de Pamplona, propone, propiciar los espacios donde los estudiantes puedan asumir su rol desde su responsabilidad como generaciones que a corto plazo deben construir sociedades donde se dé el dialogo, el respeto, la recuperación y la formación en valores ciudadanos. Se afirma que, la educación para la ciudadanía debe servir para 
reflexionar y sensibilizar sobre la convivencia y el ejercicio de la ciudadanía democrática en el centro $y$ en su entorno, lo que constituye al mismo tiempo una consideración metodológica fundamental: las formas de convivencia, el respeto, el afrontamiento no violento de los conflictos, la aceptación de la diversidad y el rechazo de cualquier forma de discriminación (Martínez, 2010).

Educandos agentes generadores de cambios transformadores de una sociedad que requiere que sean partícipes para la recuperación del tejido social, que nuestra sociedad tanto lo necesita, estos nuevos desafíos, frente a los procesos de fragmentación, diferenciación y exclusión social que padecen las sociedades dificultan la construcción de una ciudadanía plena que requiere de prácticas socioeducativas que garanticen A las personas el ejercicio de un conjunto de derechos, entre ellos el de la inclusión corporación social.

\section{Marco Teórico}

La educación en general y en este caso, la educación para la ciudadanía, representa no solo un derecho universal sino como indica Cajiao (2005), es la herramienta más eficaz de la que disponen los pueblos para combatir la desigualdad, la pobreza, la privación de los derechos humanos y avanzar hacia sociedades más democráticas e igualitarias. Así mismo quedó inscrita en la Declaración Universal de los Derechos Humanos Organización de las Naciones Unidas (1948):

La educación ha de tener por objeto el pleno desarrollo de la personalidad humana y el fortalecimiento del respeto a los derechos humanos $\mathrm{y}$ de las libertades fundamentales; favoreciendo la comprensión, la tolerancia y la amistad entre todas las naciones y todos los grupos étnicos y religiosos. (Art.26)

En su carácter universal tiene como finalidad instrumental la movilidad y nivelación social: los individuos logran agenciar sus vidas a través de ella y la sociedad avanza en materia científica e invención técnica y tecnológica que permite cerrar brechas y como finalidad intrínseca: dota a los seres humanos de capacidades que van desde las libertades políticas hasta la autonomía para vivir de acuerdo a razones que valoren la vida que sean vivir.

Es decir, ésta es la vía por la cual tanto los pueblos como los individuos pueden ver realizadas sus aspiraciones: materiales, políticas y espirituales, de allí a que no solo tenga un vínculo directo con la democracia y sino que sea a través de ella que se fortalezcan los regímenes democráticos y eso implica necesariamente formar subjetividades políticas, ya que como plantea Freire (2011), la ciudadanía es un proceso inacabado, jamás termina, somos ciudadanos durante toda la vida y eso exige formar ciudadanía con claridad y coherencia política, en y para la democracia. En ese sentido, si la pretensión es formar jóvenes, hombres y mujeres con conciencia sobre la realidad del mundo y su momento histórico, entonces debe hacerse desde una perspectiva crítica, curiosa, que interrogue, cuestione y genere asombro y eso implica un ejercicio político y que así mismo se conciba la escuela y toda práctica que en ella se desarrolle, como práctica política. Las finalidades de la pedagogía crítica.

Por otra parte, señala Nussbaum (2012) que existe una relación estrecha entre desarrollo humano y democracia: si queremos participar en las decisiones que regirán los destinos de nuestra sociedad y sus trasformaciones sociales debemos tener voz y voto en los espacios públicos donde se toman dichas decisiones y solo en una democracia podría hacerse, algo que además garantiza la dignidad y reivindica el ejercicio y la participación ciudadana, tal como indica Sen (2006) la noción de democracia va mucho más allá de un proceso electoral donde las personas por medio del voto eligen quien los representa ante el poder público, esta es mucho más valiosa "incluye la posibilidad de que los ciudadanos participen en el debate político y, con ello, de estar en disposición de influir en las opciones relativas a los asuntos públicos" (p.12). 
Cuando el autor afirma que la visión democrática es mucho más amplia permite incluir en el debate que debemos formar ciudadanos y ciudadanas no solo para votar sino para dirigir también las riendas de la sociedad y controvertir aquellas decisiones autoritarias y antidemocráticas, donde necesariamente desde el campo pedagógico debe darse importancia a la formación crítica, reflexiva y política del quehacer ciudadano. Resaltando ideas como: "la escuela ha de preparar a sus alumnos y alumnas para que sean capaces de asumir en el futuro las ideas y hábitos democráticos" (Rovira, 1996, p.28).

Esta última consigna entra a cuestionar $\mathrm{e}$ inquietar frente a la perspectiva pedagógica desde donde se está formando al ciudadano y la ciudadana que ha de ser protagonista de la vida pública y escribir los reglones de su propia historia en su paso por la agencia social y política como adulto demócrata.

Desde esas miradas, puede afirmarse que una sociedad es democrática en la medida que se prepara a sus gentes para la participación real de sus bienes y las instituciones son recreadas en función de ello, como la escuela por ejemplo, ya que es aquí donde se llevarán a cabo dichas interacciones y como establece Dewey (1953) "tal sociedad debe tener un tipo de educación que dé a los individuos un interés personal en las relaciones y el control social y los hábitos espirituales que produzcan los cambios sociales sin introducir el desorden" (p.108), permitiéndonos entender la necesidad de formar sujetos plenamente libres: un sujeto que tenga la posibilidad de orientarse hacia un objetivo de forma autónoma, de tomar sus propias decisiones sin verse constreñido por la voluntad de otros. En palabras de Freire (2008), una educción para la libertad o la educación como práctica de la liberad.

Por su parte plantea Giroux (2012), "las escuelas figuran entre los pocos espacios de la vida pública en los que los estudiantes, jóvenes o viejos, pueden experimentar y aprender el lenguaje de la comunidad y de la vida pública democrática" (p.14). Con ellos se plantea que el lugar idóneo para la construcción del sujeto democrático es la escuela, el sujeto político que es participe y autor, al tiempo, de las modificaciones que la sociedad amerita, la vigencia de sus luchas, que no es otra cosa que como indica Giroux (2006), la escuela y la lucha por la ciudadanía. Eso implica que esta, sus prácticas al interior y el ejercicio académico asuman el paradigma de la pedagogía critica en su contexto y con él se interiorice el marco político de la educación, una postura cuestionadora y reflexiva que se ejerce en el proceso de construcción y recreación del conocimiento como indica Freire (2008), y como este se convierte en fuerza social y política de lucha y emancipación.

Entonces, desde el marco de la pedagogía critica como paradigma para la formación de ciudadanías democráticas el maestro y la maestra ha de considerar que "el proceso educativo desde el contexto de la interacción comunicativa; analiza, comprende, interpreta y trasforma los problemas reales que afectan a una comunidad en particular" (Ramírez, 2008, p.109). Permitiendo desarrollar la capacidad de analizar e interpretar la realidad y junto a ella la aplicación del conocimiento reconstruido en función de transformar las problemáticas sociales concretas de su entorno, el conocimiento como fuente de liberación, si se desea utilizar términos de Freire (1970). Desde esa mirada Ramírez (2008), recrea los presupuestos teóricos de la pedagogía crítica: 1) la participación social, 2) la comunicación horizontal entre los diferentes actores que integran los estamentos, 3) la significación de los imaginarios simbólicos, 4) la humanización de los procesos educativos, 5) la contextualización del proceso educativo y 6) la transformación de la realidad social.

En ese sentido, el primer punto tiene que ver la consolidación de los valores y virtudes democráticas, la escuela ha de preparar a las y los jóvenes para la vida pública, ser visibles allí, por lo tanto, es necesario fortalecer el pensamiento político que lleva a la conciencia de la realidad y querer transformarla. Se dota de capacidades a quienes se educan para que intervengan y agencien con su voz 
y voto; la segunda postura reivindica la igualdad en condiciones para desarrollar acciones y participar plenamente en los asuntos que conciernen a todos los individuos "en este proceso los interlocutores simbolizan y significan con base en la validación del discurso del otro, con base en la legitimación de las intervenciones y análisis de las visiones que en ellas subyacen" (Ramírez, 2008, p.110).

Por su parte, el tercer aspecto indica la necesidad de retomar los procesos históricos, culturales y políticos en aras de reconstruirlos: las experiencias, los estilos de vida y las ideas con las que se creado determinada realidad en las que viven las comunidades, ya que encontrar el sentido y significado de todo ello es esencial en la formación del sujeto político consiente. La cuarta postura se inclina por recordar que el proceso educativo no es meramente un proceso de instrumentalización del conocimiento, tiene un carácter constitutivo que se fundamente en la integridad del sujeto, por eso va más allá de la simple transferencia del conocimiento: la capacidad reflexiva y analítica son fundamentales dentro del ejercicio formativo. El quinto punto toca aspectos sensibles al tejido social, una mirada no individualizadora sino comunitaria de la realidad, la comprensión de lo comunitario y nuevos horizontes que reemplacen el orden dominante que segrega y excluye y, por último, la escuela como espacio y campo político de confrontación, lucha y emancipación; en ella convergen todas las ideas, pero también todas las problemáticas y su esencia es dar cabida a las primeras y transformar las segunda.

Este breve análisis permite entender que una educación democrática y en y para la ciudadanía no debería darse al margen de la pedagogía critica, su componente ético (muy kantiano) y dialógico (muy freiriano) crean los principios de convivencia y las responsabilidades sobre las cuales debe abordarse la vida social: los pactos y contratos sociales, el respeto por las ideas y el asumir los problemas colectivas y contextuales para dar vía a la vivencia democrática. De hecho, desde esta visión de la pedagogía crítica, los y las maestras "han de contemplarse en función de los intereses ideológicos y políticos que estructuran la naturaleza del discurso, las relaciones sociales de aula y los valores que ellos mismos legitiman en su enseñanza" (Giroux, 2001, p.65), eliminado a su vez toda mirada homogenizánte, donde su riqueza radica en la valoración de las diferencias y divergencias de los sujetos.

En ese orden, la formación crítica parte de las interacciones existentes entre sujetos y realidad, no los mira como fenómenos aislados sino como consecuencias, cuestionando permanentemente ¿cómo y por qué se construye el conocimiento? al que entiende como una producción social, ¿porque este es culturalmente aceptado? y ¿qué formas de conocimiento tienen más poder que otras? Ya que se interesa en la relación conocimiento-poder y como éste valida ciertos intereses, pero al tiempo como es una herramienta de emancipación social que permite entender la manipulación de masas, el otorgamiento de privilegios y las relaciones de clases sociales. En sí, la complejidad del enfoque pedagógico critico se da en el momento que debemos entender que educar es un acto político, que la escuela es un espacio político y que el conocimiento tiene en sí mismo una finalidad política, por supuesto no partidista, ese conlleva a que como espacio político al interior de las instituciones se den luchas, formas de resistencias y rebeldías que son propias del ejercicio ciudadano y que no hay que ver como anormales sino preparar a las jóvenes para la vida adulta.

Además de lo expuesto y como complemento del mismo, es necesario reiterar que la pedagogía critica desde la perspectiva de Freire (2011) "Educación como práctica de la libertad", Freire (2012) “Pedagogía del oprimido", Freire (2011) "Pedagogía de la esperanza", Freire (2010) "Cartas a quien pretende enseñar", entre otras obras, nos invita a la reinvención de acto educativo, a desafiar los contextos e incomodar desde la pregunta intencionada que confronta y alienta, retando los discursos y las prácticas cotidianas en nuestros entornos desde el lente ético y político, ya que como la plantea Ortega (2009) "La pedagogía crítica se define como una 
instancia de formación y aprendizaje ético y político que incide en la producción de subjetividades, en los procesos de construcción y circulación de valores y en la generación de prácticas sociales" (p.28) ya que para Giroux citado por González (2006), esta ve la educación como "una práctica política social y cultural, a la vez que se plantea como objetivos centrales el cuestionamiento de las formas de subordinación que crean inequidades, el rechazo a las relaciones de salón de clases que descartan la diferencia y el rechazo a la subordinación del propósito de la escolarización a consideraciones económicas" (p. 83).

Volviendo a Freire (2011), contrario a la educación bancaria que controla el pensamiento y las acciones de los individuos, instaurando la incapacidad crear, recrear y actuar libremente, la frustración y la opresión; la pedagogía liberadora como practica de liberación promueve la idea de que debemos ser conscientes de nuestra relación con el mundo y romper con los hábitos que nos domestican, reconquistar el lenguaje y tener voz en los espacios donde nos ubicamos y participamos de la vida social y política. El quehacer pedagógico en ese sentido es educar para transmutar la sociedad oprimida, hacer visible lo oculto, despertar el espíritu ingenioso y creador del ser humano, moldeado la cultura y la dominación tradicional. Lo anterior permite eso que el autor denomina la transitividad crítica, entendido esta como la capacidad de pasar de una forma de entender el mundo y la realidad a otra incentivada por la razón, producto de una educción dialogal y activa y que se ubica en una responsabilidad social y política que profundiza en la interpretación de los problemas.

Siguiendo con Freire (2012), su puesta en marcha provoca la compresión de nuevos desafíos que van permitiendo descubrir el mundo comprometiéndose en la praxis con su transformación, ya que la educación problematizadora rehace constantemente su objeto donde las y los educando van viviendo una metamorfosis de individuos dóciles completamente receptores a sujetos críticos en pleno dialogo e investigadores. En sí:

La educación como práctica de la libertad, al contario de aquella que es práctica de la dominación, implica la negación del hombre en abstracto, aislado, suelto, desligado del mundo, así como la negación del mundo como una realidad ausente de los hombres. (Freire, 2012, p.87)

Otra autoridad al respecto es Giroux (2006), sobre todo en su obra la escuela y la lucha por la ciudadanía, donde comprende que uno de los grandes retos del quehacer educativo es:

Desarrollar una forma de ciudadanía en la que el lenguaje público haga suya, como referente para la acción, la eliminación de aquellas condiciones ideológicas y materiales que fomentan diversos modos de subyugación, segregación, brutalidad y marginación, frecuentemente expresadas por medio de formas sociales que encarnan intereses raciales, clasistas y sexistas. (p.22)

Es decir es necesario romper todas las formas de dominación y opresión, tal cual como lo referencia Echeverry (2011), formar el sujeto democrático inscribe la idea despertar en cada individuo el aceptar que hace parte de una sociedad pluralista; prepararlo para la relación con el otro y con los otros; para que aprenda a reconocer quién es, cómo vive, cómo piensa, cómo trabaja, qué sueños y aspiraciones tiene, qué dificultades lo marginan y lo excluyen, qué oportunidades tiene.

En ese orden, Giroux (2003) propone algunas consideraciones teóricas para el desarrollo de una teoría crítica de la educación cívica, considera de vital importancia para una política y pedagogía de la ciudadanía critica re-hacer un lenguaje visionario y una filosofía pública que coloquen a la igualdad, la libertad y la vida humana en el centro de las categorías de democracia y ciudadanía, teniendo en cuenta que la democracia es un escenario de lucha $\mathrm{y}$, como practica social, impulsa la adquisición 
de conceptos ideológicos como poder, política y comunidad, los que se hallan en permanente contradicción. Una ciudadanía activa no limita los derechos democráticos a la participación de las urnas electorales, por el contrario, su alcance es mayor, abarca otros escenarios de participación como la economía y el Estado.

Así mismo, Giroux (2003) asevera que "las escuelas, consideradas como esferas publicas democráticas, centran sus actividades en la indagación crítica y el diálogo significativo. En este sentido, los estudiantes tienen la posibilidad de aprender el discurso de la asociación pública y de la responsabilidad ciudadana" (p.86). Su discurso intenta concebir la idea de una democracia crítica que exige respeto por la libertad individual y por la justicia social, una vez concebido éste, las escuelas se pueden preservar como instituciones que proveen conocimientos, habilidades y relaciones sociales, premisas básicas para educar a ciudadanos capaces de construir una democracia crítica. Este autor asegura que la esencia de la enseñanza pública requiere que los docentes no solo dominen la materia que van a enseñar, igualmente deben tener conocimientos básicos de la naturaleza económica, política y cultural de la propia enseñanza escolar. En ese sentido requieren aprender un lenguaje pluridisciplinario que integre la historia, la sociología, la filosofía, la economía política y la ciencia política de la enseñanza escolar.

En esa misma línea se inscribe McLaren (2005), con su obra La vida en las escuelas, desde donde plantea la postura deunapedagogía revolucionaria" $\mathrm{La}$ pedagogía crítica evita todo enfoque de la pedagogía que la redujera a la enseñanza de habilidades de miras estrechas y aisladas de los debates y contextos de discusión en los que se las utiliza" (p.74) y afirma desde esa mirada, que una de las grandes premisas que debe tener en cuenta el quehacer pedagógico es que todos los pensamientos, actos y relaciones son políticos en su sentido ideológico, por lo tanto la formación política no puede ni debe convertirse en un ejercicio instrumental. Como bien lo expresa
Ortega (2009), "La práctica pedagógica se constituye en una práctica intencionada; por consiguiente, es una práctica orientada por fines. Es, entonces, una práctica ética y, por consiguiente, también política" (p.31). Dando sentido a todo lo que se ha expuesto hasta aquí.

Por su parte, para Habermas (1999) en la interpretación republicana de la democracia, la ciudadanía se realiza fundamentalmente en las prácticas colectivas de autodeterminación, cuando los ciudadanos ejercen la soberanía y se dan sus propias normas de convivencia. El modelo republicano de ciudadanía recuerda que las instituciones de la libertad garantizadas jurídicamente sólo tienen el valor que les conceda una población habituada a la libertad política y acostumbrada a la perspectiva del «nosotros» propia de las prácticas autogestionarias. En la perspectiva republicana, la ciudadanía es concebida según el modelo de la participación en una comunidad ético-cultural que se autodetermina en todo aquello que tiene implicaciones con el bien común. Los ciudadanos se integran en la comunidad política como las partes en el todo, de manera que sólo pueden construir su identidad personal y social bajo el horizonte de las tradiciones comunes y de las instituciones políticas reconocidas.

Establecido lo anterior, a continuación se presenta un análisis de las investigaciones revisadas, las cuales se agrupan teniendo en cuenta los países de origen y las categorías emergentes de estos análisis relacionados con el tema de la construcción de ciudadanía.

Según Clarke (2010) sostiene que las aulas y escuelas transformadoras son las que permiten la formación de ciudadanos integrales, conscientes de actuar en un mundo que comparte con otros, conscientes de la relación que existe entre su propia identidad y la de los otros, y comprometidos con el mundo.

A lo anterior, Lawy \& Biesta (2006), agregan un factor condicionante, la noción de ciudadanía 
relacionada directamente a la política oficial. Aunque no apropiado, consienten en que se presume que los jóvenes deben comportarse de cierta forma para lograr el estatus de ciudadanos. A esta mirada de la formación en ciudadanía la denominan ciudadaníacomo-logro.

Por consiguiente, Delli Carpini \& Keeter (1996) sostienen que ciudadanos informados han demostrado ser mejores ciudadanos según los estándares de teoría y práctica democrática que sustentan el sistema americano. Éstos tienen mayores probabilidades de participar en política, de tener actitudes estables y significativas en ciertos temas, son capaces de unir sus intereses con sus actitudes, de elegir candidatos que sean consistentes con sus actitudes, son capaces de apoyar normas democráticas como extender libertades básicas civiles a miembros de grupos no populares.

\section{Intervenciones pedagógicas en la ética ciudadana y la cultura democrática.}

La evolución del concepto de ciudadanía en un mundo dinámico y cambiante como el nuestro, ha dependido casi por completo del momento históricopolítico en que se registra. De igual manera, a ésta hay que entenderla como la posición e interrelación libre del individuo con edad y derecho a ejercer o gozar de facultades civiles, sociales e institucionales en su comunidad y con sus semejantes. Por su parte, como materialización de lo anterior, es ciudadano aquella persona que nace o reside por cierto tiempo en una sociedad organizada y es titular de derechos y deberes que debe ejercer o acatar. Sin embargo, es de advertir que estos conceptos básicos per se no revelan la complejidad del concepto de ciudadanía que se ha desarrollado en los modernos EstadosNación.

A manera de conclusión, tres aspectos rigen todo este plan temiendo: la educación como vía o vehículo de nivelación y movilización social hacia sociedades más justas y democráticas, la ciudadanía como un ejercicio cultivado en las escuelas para garantizar la conciencia sobre la realidad y la autonomía de los individuos para participar en ella y la pedagogía crítica como paradigma y referente sobre el cual recae la formación del sujeto político dotado de derechos y denominado ciudadano o ciudadana. Siendo el punto central el papel y la importancia de la pedagogía critica desde una perspectiva democrática y transformadora. Que permite recoger varios postulados para la reingeniería de la escuela y sus prácticas.

\section{Metodología}

El presente capitulo muestra el diseño metodológico que caracterizará la investigación, corriente teórica, tipo y enfoques utilizados, técnicas de recolección.

\section{Enfoque}

Para González (2013), la investigación cualitativa tiene como propósito la construcción de conocimiento sobre la realidad social, a partir de las condiciones particulares y la perspectiva de quienes la originan y la viven; por tanto, metodológicamente implica asumir un carácter dialógico en las creencias, mentalidades y sentimientos, que se consideran elementos de análisis en el proceso de producción y desarrollo del conocimiento con respecto a la realidad del hombre en la sociedad de la que forma parte.

Por otro lado, Según Jiménez (2000) los métodos cualitativos parten del supuesto básico de que el mundo social está construido de significados y símbolos. De ahí que la intersubjetividad sea una pieza clave de la investigación cualitativa y punto de partida para captar reflexivamente los significados sociales. La realidad social así vista está hecha de significados compartidos de manera intersubjetiva. El objetivo y lo objetivo es el sentido intersubjetivo que se atribuye a una acción. La investigación cualitativa puede ser vista como el intento de obtener una comprensión profunda de los significados y definiciones de la situación tal como nos la presentan 
las personas, más que la producción de una medida cuantitativa de sus características o conducta.

En esta investigación se muestra la aplicación de un enfoque cualitativo para trabajar los aspectos de pedagogía crítica y formación ciudadana, siendo fundamental para la mejora en la percepción de un mejor ambiente de la institución y el entorno social que se desenvuelva. Este trabajo hace énfasis en lograr mejores prácticas en el manejo y pensamiento de las diferentes situaciones en la que se encuentre.

\section{Diseño de la investigación}

De acuerdo con Malhotra (1997) el diseño de la investigación es una estructura o un plano que sirve para dirigir un proyecto de investigación. Permite detallar los pasos necesarios para obtener información indispensable en la solución al problema que nos ocupa. Aunque exista un planteamiento amplio del problema, el diseño de la investigación, específica los detalles para determinar este. "Un buen diseño de la investigación, asegura que el proyecto se realizará de manera efectiva y eficiente" (Malhotra, 1997, p.86) generalmente el diseño de la investigación incluye los siguientes pasos:

1. Definir la información necesaria a través de los fundamentos teóricos principales, para determinar los principales postulados de la pedagogía crítica y su influencia en la formación ciudadana en los estudiantes de pedagogía infantil.

2. Diseñar las fases exploratorias, descriptivas o causales. Para indagar los elementos necesarios en el estado en que se encuentran los estudiantes y maestros del programa de pedagogía infantil, esto en una fase exploratoria; Describir el proceso de desarrollo de la formación ciudadana identificando las características de las categorías y subcategorías a observar, para comprender cuales son los posibles aportes al proceso educativo, a través del dialogo y debate con la población objeto de estudio. La claridad de las dos principales categorías y sus correspondientes subcategorías permite continuar con la siguiente fase.

3. Construir y probar previamente una entrevista o una forma apropiada para recolectar datos recabados. Se establecen una serie de Categorías y Subcategorías que permiten conocer cuáles son los conocimientos de los estudiantes y maestros para la elaboración de preguntas en torno a las categorías (Pedagogía Critica, Ciudadanía y Formación ciudadana) este proceso permitirá identificar las percepciones del pensar de la población objeto de estudio, la información se completa con el desarrollo de unas actividades en las cuales mediante talleres expondrán los conocimientos de la formación ciudadana.

\section{Como observa la Pocovi (2005):}

La entrevista online es definida como la comunicación $\mathrm{y} / \mathrm{o}$ diálogo entre el sujeto $\mathrm{y}$ el investigador a través de la medición de la computadora, haciendo uso de recursos como el chat, el email, y/o foros virtuales, a través de los cuales se puede propiciar una interacción secuencial, planeada y organizada en un periodo concreto y con un propósito especifico determinado por las necesidades de información de la investigación. (p.15)

Este modelo fue el más conveniente a la hora de aplicar la propuesta metodológica para verificar los niveles de apropiación de los aprendizajes de la formación ciudadana en los estudiantes del programa de pedagogía infantil, ampliación Cúcuta.

4. Especificar el proceso de muestreo y el tamaño de la muestra. La toma de muestra y análisis de los resultados se elabora con docentes en una primera instancia que han impartido el curso de formación ciudadana y del modelo de la pedagogía critica, en el desarrollo de los contenidos programáticos de la línea de la formación socio humanística en el pensum académico del programa de Licenciatura en Pedagogía Infantil, por otra parte los estudiantes 
de séptimo y octavo semestre en una población de 8 estudiantes quienes han completado un $70 \%$ del pensum académico del programa.

5. Desarrollar un plan para el análisis de los datos recabados, la información que se retome en una primera instancia se tendrá como principal referencia las categorías y subcategorías más pertinentes de los temas, se elabora un instrumento tipo entrevista para recolectar las respuestas concretas, luego se elabora un análisis pregunta por pregunta entre las diferentes reacciones de los docentes y estudiantes. Debido a que es un proceso muy personal, no se trabajó con una definición precisa, aunque se aclara la importancia que se le da a la información obtenida en la entrevista vía ZOOM, además de cómo se procesa en el cuadro de preguntas y respuestas de entrevistados. Cada una de las respuestas de los entrevistados sirvió para construir algunas ideas generales, que se verán en el análisis de los resultados.

\section{Guía de entrevista para los estudiantes.}

Primero se realizará el acercamiento a la concepción que tenía cada uno de los participantes sobre la formación de ciudadanía. Existen estudios al respecto de la ciudadanía se encuentra varios libros y tesis sobre el tema y extensa bibliografía. Para llegar a la elaboración de lineamientos se ve la necesidad de iniciar con la claridad conceptuales al respecto, por ello fue necesario realizar la indagación desde la comunidad educativa sobre esta concepción haciendo la pregunta ¿qué es para usted la ciudadanía?, que entiende por formar en ciudadanía? De igual manera se pretende indagar la concepción de ciudadanía a través de la entrevista, que se aplicarán previamente estudiantes y docentes, con las preguntas: ¿En dónde se evidencia la ciudadanía a través de su formación en el ser?, ¿cómo se evidencia la ciudadanía en su quehacer?

En un segundo momento se buscará por las creencias sobre la formación en ciudadanía preguntas que tocan de manera individual la praxis, las experiencias, estrategias y espacios que originan el proceso de este ejercicio entre los docentes y estudiantes de programa de pedagogía infantil de la Universidad de Pamplona, los que nos darán la rutas para construir los lineamientos, las preguntas que nos permitieron ahondar en este aspecto fueron: ¿Cómo cree usted se debe formar en ciudadanía? ¿Qué estrategias pedagógicas cree usted promueve la ciudadanía? ¿Qué prácticas favorecen el ejercicio de la ciudadanía en la Universidad de Pamplona? ¿Qué espacios, cree usted, propicia la Universidad de Pamplona para formar en ciudadanía? ¿Qué hechos crees pueden impedir que se ejerza la ciudadanía? ¿Crees que tus estudiantes adscritos de la licenciatura de pedagogía infantil vivencian la ciudadanía?

En el proceso de la realización de las entrevistas a profundidad se ahondarán en los aspectos particulares que emergían de la misma y enriquecieron cada una de las categorías y subcategorías. Erickson (1982) sustenta que la característica primordial de la investigación cualitativa es el lugar central que ocupa la interpretación, y que los resultados de la investigación no son tanto "descubrimientos" como "afirmaciones" (p.46).

\section{Categorías conceptuales, dimensiones $\mathbf{y}$ subdimensiones de las categorías conceptuales}

La política institucional frente a la formación en ciudadanía autónoma a partir de las concepciones de la comunidad académica. Para la Universidad de Pamplona, la formación integral debe contribuir de manera efectiva a enriquecer el proceso de socialización del estudiante desde una perspectiva analítica y crítica, afinar su sensibilidad mediante el desarrollo de sus valores estéticos y, fortalecer su responsabilidad a través de la definición o determinación de sus compromisos consigo mismo y con la sociedad.

Consecuente con lo anterior, el Pensamiento Pedagógico Institucional de la Universidad de Pamplona, expresa los principios y lineamientos generales que orientan el quehacer académico universitario, para cumplir de manera adecuada 
e innovadora con el desarrollo de los procesos de formación integra a los que se ha comprometido la institución en su misión y visión. Ante todo se formula la misión institucional:

La Universidad de Pamplona, en su carácter público y autónomo, suscribe y asume la formación integral e innovadora de sus estudiantes, derivada de la investigación como práctica central, articulada a la generación de conocimientos, en los campos de las ciencias, las tecnologías, las artes y las humanidades, con responsabilidad social y ambiental. (Universidad de Pamplona, 2012; Ley 115 de 1994, p.1-50)

\section{Las categorías de análisis que se visualizan desde los documentos de política institucional son las siguientes:}

Desde los estudiantes el pensamiento crítico, bien común y comunicación. La categoría pensamiento crítico, vislumbra las subcategorías: Sujeto crítico, enseñar a pensar y empoderamiento. En la categoría bien común las subcategorías Compromiso social, Formación integral, Libertad, Participación, Proyección comunitaria y la categoría comunicación: Formación ético dialógica y trabajo colaborativo.

Tabla 1. Categorías generales y preguntas

\begin{tabular}{|c|c|}
\hline $\begin{array}{l}\text { Categorías } \\
\text { generales }\end{array}$ & Preguntas \\
\hline $\begin{array}{l}\text { Pedagogía } \\
\text { Critica }\end{array}$ & $\begin{array}{l}\text { ¿Cómo puede definir el concepto de pedagogía crítica? ¿Describir? } \\
\text { ¿Bajo qué referente teóricos fundamenta la formación en pedagogía } \\
\text { crítica? } \\
\text { ¿Qué vínculo determina que existe una relación entre pedagogía crítica } \\
\text { y formación para la ciudadanía? }\end{array}$ \\
\hline Ciudadanía & $\begin{array}{l}\text { ¿Para usted Qué es ser ciudadano o ciudadana? } \\
\text { ¿Qué es la ciudadanía? } \\
\text { ¿Cuándo se ejerce la ciudadanía? } \\
\text { ¿Cuál es la obligación del ciudadano? } \\
\text { ¿Qué factores no permiten el ejercicio de la ciudadanía? } \\
\text { ¿Conoce cuáles son los derechos fundamentales del ciudadano } \\
\text { colombiano? } \\
\text { ¿Por qué formar en ciudadanía? } \\
\text { ¿Cuándo se forma en ciudadanía? } \\
\text { ¿Cómo se lleva a cabo la formación ciudadana al interior del programa } \\
\text { de Pedagogía Infantil? ¿Qué cursos, contenidos... que la propician? } \\
\text { ¿Qué estrategias y prácticas que desarrolla en su ejercicio académico } \\
\text { promueven la formación ciudanía? }\end{array}$ \\
\hline $\begin{array}{l}\text { Formación } \\
\text { ciudadana }\end{array}$ & $\begin{array}{l}\text { ¿Qué prácticas favorecen el ejercicio de la ciudadanía en la } \\
\text { universidad? } \\
\text { ¿Qué espacios propicia el programa de licenciatura en Pedagogía } \\
\text { Infantil para formar en ciudadanía? }\end{array}$ \\
\hline
\end{tabular}


Tabla 2. Categorías y subcategorías sustentadas por la población participante y sujetos claves en la investigación sobre la pedagogía crítica en la formación ciudadana en estudiantes universitarios: caso Universidad de Pamplona programa licenciatura en pedagogía infantil, sede Cúcuta

\begin{tabular}{ll}
\hline Categorías generales & \multicolumn{1}{c}{ Subcategorías } \\
\hline \multirow{2}{*}{ Pedagogía critica } & Sujeto critico \\
& Enseñar a pensar \\
& Empoderamiento \\
& Compromiso Social \\
& Decidir con argumento \\
& Libertad \\
& Participación \\
& Bien de la comunidad \\
& Compromiso social \\
Formación Ciudadana & Decidir con argumentos \\
& Formación integral \\
\hline
\end{tabular}

\section{Resultados y Discusiones}

La presente investigación se llevó a cabo en la Universidad de Pamplona específicamente con los estudiantes de pregrado de 8 y 9 semestre pertenecientes a la licenciatura de pedagogía infantil los cuales son formados como futuros profesionales en educación de la infancia, sólidamente formado a través de un currículum que responde pertinentemente a los nuevos escenarios que los procesos de transformación en materias de calidad demandan a la Educación, junto con el compromiso de formar ciudadanos críticos que permitan reconocer las problemáticas sociales de su entorno y de esa manera analizar y contribuir en la generación de soluciones reales; el futuro profesional está formado para dominar conceptualmente el campo de las ciencias de la educación para desenvolverse con calidad en su praxis profesional, así como desarrollarse con seguridad y confianza en sus competencias comunicativas, argumentativas, propositivas, interpretativas, instrumentales, procedimentales, laborales y actitudinales. Además, que será un ciudadano competente que comprenda la empatía social para saber actuar en la toma de decisiones.

El licenciado en pedagogía infantil, es el encargado de detectar y dar solución a los problemas educativos, así como realizar investigación formativa que aporten transformaciones a esos mismos procesos. Posee habilidades interpersonales, cognitivas, de análisis y de comprensión de contenidos para descubrir relaciones causales que le permiten vislumbrar las problemáticas educativas presentes y futuras.

La información recaudada de esta investigación se realizó a través de las entrevistas Semiestructuradas y el grupo focal, se analizó a través del ATLAS.TI, el principal soporte informático para desarrollar TF, este programa fue diseñado a finales de los ochenta por el alemán Thomas Murh, quien recurriendo a la tecnología hizo un intento por aplicar los planteamientos metodológicos de Glaser \& Strauss (2002).

A través del software Atlas. Ti el cual permitió organizar la información ingresando los datos arrojando los resultados de la entrevista semiestructurada aplicada a los sujetos claves; es decir a través de las respuestas que nos aportaron la población participante o sujetos claves.

Este software permite expresar el sentido circular del análisis cualitativo, por cuanto otorga la posibilidad de incorporar secuencialmente los datos, sin la necesidad de recoger todo el material en un mismo tiempo. Por esta razón, permite llevar a cabo el muestreo teórico necesario para realizar el análisis constructor de teoría". 
El análisis de la información se hace las diversas fuentes que brindan un soporte de tipo metodológico pero definitivo en la configuración de la investigación: la interrelación categorial y subcategoría que surge de la codificación de la información aportada por los profesores al interior de cada categoría y subcategoría analizada, la interpretación del investigador, el marco referencia y la codificación selectiva derivada del enfoque cualitativo en razón a que como lo señalan Strauss $\&$ Corbin (2002), se hace referencia a un "proceso no matemático de interpretación, realizado con el propósito de descubrir, conceptos y re relaciones en los datos brutos y luego organizarlos en un esquema interpretativo teórico" (p.13).

De allí que los componentes de la investigación cualitativa sean los datos, los procedimientos para interpretar y organizar los datos y los informes escritos y verbales. "Al hecho de conceptualizar, reducir, elaborar y relacionar los datos se lo suele denominar codificar" (Strauss \& Corbin, 2002, p.13).

En esta investigación, las categorías de análisis fueron emergiendo a medida que se avanzaba en la revisión teórica recolectada y de los datos suministrados, en este caso, las entrevistas de los docentes. La construcción de las categorías pasa necesariamente por la codificación de la información, tal codificación es según Strauss \& Corbin (2002) un "proceso analítico mediante el cual se identifican los conceptos y se descubren los datos, sus propiedades y dimensiones" (p.110). Para facilitar la organización de los datos, se presenta a continuación la relación de las categorías Cuadro 1. Categorías y preguntas orientadoras de la entrevista en profundidad a los Docentes y Estudiantes del programa de Pedagogía Infantil de la universidad de pamplona. Para ello, la triangulación que se desarrolló incluye la teoría, resultados del método descriptivo y hermenéutico de los informantes y fuentes de recolección de datos.

La triangulación será un proceso permanente en el que la información y su respectivo análisis para elaborar un constructo teórico, que corresponde al objetivo esencial del presente proyecto. La codificación será selectiva, porque permitirá integrar las categorías alrededor de una sola, validar las relaciones y completarlas. Al respecto Strauss \& Corbin (2002), afirman, "No obstante, sólo cuando las categorías principales se integran finalmente para formar un esquema teórico mayor, los hallazgos de la investigación adquieren la forma de teoría. La codificación selectiva es el proceso de integrar y refinar las categorías" (p.157).

Se puede inferir, que, en el presente trabajo heurístico, el investigador va a reducir datos aportados por los Docentes y Estudiantes estos conceptos derivados de la interpretación y aplicación de la ley 115 de /94 donde se evidenciará en la formación pedagógica sobre el conocimiento de los derechos humanos en un conjunto de afirmaciones, que servirán para explicar una teoría, ya generalizada, producto de la opinión de los sujetos entrevistados. Según Uribe, Zapata \& Gómez (2002)

Cuanto más válidas, confiables y representativas sean estas informaciones, mayor será la posibilidad de reorganizar los fines y los medios de un programa o proyecto, de tal manera que éste arroje resultados óptimos. Obtener y valorar estas informaciones es lo que denominamos evaluación. (p.29)

\section{Conclusiones}

En la presente investigación estuvo respaldada por el estudio descriptivo, ya que es un proceso sistematizado de analizar las concepciones que tienen los estudiantes del la Universidad de Pamplona sede Cúcuta, de la formación ciudadana acuerdo a las experiencias vividas absteniéndose de formular juicios de cualquier clase y centrándose en reflexionar y describir el tema central. Es así como se presentaron los principales hallazgos del proceso investigativo organizados en tres categorías competencias ciudadanas, Pedagogía crítica, Ciudadanía y formación ciudadana. 
En ese sentido, se puede concluir de acuerdo a los resultados obtenidos es que inicialmente el modelo utilizado por los docentes que se encargan de impartir la materia de cívica, está basado adecuadamente con la formación ciudadana según los lineamientos del Ministerio de Educación Nacional. Ya que los estudiantes solo tienen conocimiento del área de cívica y se les dificulta reconocer el concepto de ciudadanía

Como consecuencia los estudiantes no fomentan actitudes de participación ya que no se generan los suficientes espacios que vayan acorde con los alumnos, espacios que les permitan fortalecer las relaciones humanas ya que ellos están en la etapa donde solo quieren el reconocimiento de sus iguales, de sus compañeros, están en la etapa en la que no miden sus acciones y las consecuencias que les puedan traer. Por eso es importante escuchar sus necesidades y hacerlos parte de la construcción de la ciudadanía tal como lo requiere la sociedad de hoy.

Otro punto importante es que la universidad cuenta con programas de estudio como especialización, maestrías y un personal docente que permita fortalecer estas temáticas trabajadas en el presente proyecto.

\section{Referencias}

Cajiao, F. (2005). Enseñanza aprendizaje y deserción escolar. Revista economía colombiana y coyuntura política, 311(5), 1-12.

Clarke, P. (2010). Ser ciudadano y praxis. Madrid: Editorial Sequitur.

Delli, M. \& Keeter, S. (1996). What americans know about politics and Why it matters. New Haven: Yale University Press

Dewey, J. (1953). Democracia y Educación. Una introducción a la filosofía de la educación. Buenos Aires: Losada.

Echeverry, M. (2011). Formación ciudadana y escuela Una mirada desde la ciudadanía democrática. Cali: Universidad del Valle.

Erickson, F (1982). La investigación en la enseñanza. Barcelona: Paidós.

Freire, P. (1970). Pedagogia do oprimido. Rio de Janeiro: Paz e Terra.

Freire, P. (2008). Pedagogía de la autonomía. Siglo XXI: México.

Freire, P. (2010). Cartas a quien pretende enseñar. Siglo XXI: México.

Freire, P. (2011). La educación como práctica de la libertad. México: Siglo XXI.

Freire, P. (2011). Pedagogía de la esperanza, un reencuentro con la pedagogía del oprimido. Siglo XXI: México.

Freire, P. (2012). Pedagogía del Oprimido. Siglo XXI: Buenos Aires.

Giroux, H. (2001). Los profesores como intelectuales transformativos. Revista Docencia, 5(12), 60-66.

Giroux, H. (2003). Pedagogía y política de la esperanza. Teoría, cultura y enseñanza. Buenos Aires: Amorrortu.

Giroux, H. (2006). La escuela y la lucha por la ciudadanía. México, Siglo XXI.

Giroux, H. (2012). Metodología de las ciencias humanas. México: Fondo de Cultura Económica.

Gonzales, E. (2016). Acerca del estado de la cuestión o sobre un pasado reciente en la investigación cualitativa con enfoque hermenéutico. Unipluriversidad, 13(1), 60- 63.

Habermas, J. (1999). La Inclusión del Otro. Barcelona: Paidós 
Jiménez, B. (2000).Investigación cualitativa y psicología social crítica. Contra la lógica binaria y la ilusión de la pureza. Investigación cualitativa en Salud, 45(1), 1-12. Recuperado de: http://www. cge.udg. mx/revistaudg/rug17/3invesigacion. $\mathrm{html}$

Lawy, S. \& Biesta, J. (2006). Citizenship-as-practice: the educational implications of an inclusive and relational understanding of citizenship. British Journal of Educational Studies, 54(1), 34-50.

Ley 115 de 1994. Por la cual se expide la ley general de educación. Bogota: Diario Oficial.

Malhotra, N, (1997). Investigación de mercados un enfoque práctico. Barcelona: Prentice Hall.

Martínez, M. (2001). El contrato moral del profesorado. Condiciones para una nueva escuela. Bilbao: Desclée de Brouwer.

McLaren, P. (2005). La vida en las escuelas: una introducción a la pedagogía crítica en los fundamentos de la educación. México: Siglo XXI.

Nussbaum, M. (2012). Sin fines de lucro: por qué la democracia necesita de las humanidades. Bogotá: Katz.

Organización de las Naciones Unidas. (1948). Declaración Universal de los Derechos Humanos, (Art.26). Recuperado de: https://dudh. es/26/

Ortega, P. (2009). La Pedagogía Crítica: Reflexiones en torno a sus prácticas y sus desafíos. Pedagogía y Saberes, 31(4), 22-35.

Pocovi, P. (2005). La entrevista y los medios electrónicos. Recuperado de: http://imgbiblio. vaneduc.edu.ar/fulltext/files/TC113921.pdf manera ética de generar procesos educativos. Revista Folios, 28(1), 108-119.

Rovira, J. (1996). Cómo fomentar la participación en la escuela. Serie Pedagogía: Teoría y Práctica. Barcelona: Grao.

Sen, A. (2000). Desarrollo y libertad. Barcelona: Planeta.

Strauss, A. \& Corbin, J. (2002). investigación cualitativa. Técnicas y procedimientos para desarrollar la teoría fundamentada. Antioquia: Universidad de Antoquia.

Universidad de Pamplona. (2012). Actualización PEI y pensamiento pedagógico 2012. Recuperado de: http://www.unipamplona.edu.co/actualizacion_ pei.jsp

Uribe, S., Zapata, A. \& Gómez, B. (2002). Investigación evaluativa. Bogota: Instituto Colombiano para el Fomento de la Educación Superior.

Ramírez, R. (2008). La pedagogía crítica Una 